\title{
Potencial uso dos resíduos de conchas de moluscos: uma revisão
}

\author{
Potential use of shellfish waste: a review \\ Uso potencial de los desechos de mariscos: una revisión
}

Recebido: 06/02/2022 | Revisado: 12/02/2022 | Aceito: 20/02/2022 | Publicado: 02/03/2022

\author{
Tainã Fabiane da Silva Fagundes \\ ORCID: https://orcid.org/0000-0001-8749-8400 \\ Universidade Federal da Paraíba, Brasil \\ E-mail: tainaa.fagundes@hotmail.com \\ Lucineide Balbino da Silva \\ ORCID: https://orcid.org/0000-0002-8550-080x \\ Universidade Federal da Paraíba, Brasil \\ E-mail: lucineide@ct.ufpb.br
}

\begin{abstract}
Resumo
O Brasil é um grande produtor de moluscos bivalves da América Latina. A atividade de malacocultura é reconhecida mundialmente como uma importante alternativa de geração de empregos, renda e alimento, que tem contribuído para a fixação de comunidades tradicionais em seus locais de origem de modo mais sustentável. Porém, as densidades elevadas de moluscos bivalves produzidas pelo cultivo podem causar impactos ambientais provocando desequilíbrios nos ecossistemas, devido a uma maior produção dos resíduos sólidos das conchas, os quais se originam a partir da retirada dos moluscos dos interiores das conchas. O objetivo deste artigo é apresentar uma revisão bibliográfica realizada a partir da literatura vigente sobre a estrutura morfológica da concha de molusco e as possíveis aplicações dos seus resíduos. A estrutura externa da concha é colorida (perióstraco), composta por uma camada intermediária com placas de aragonita comprimidas com espessura de cerca de $0,5 \mu \mathrm{m}$, incorporada em uma fina matriz orgânica e por outra calcificada contendo agulhas de calcita de vários comprimentos e diâmetros, e por uma camada mais interna nacarada também calcária. As conchas de moluscos são constituídas principalmente por carbonato de cálcio (CaCO3). Por isso, os resíduos da concha têm sido utilizados na fabricação de blocos e pavimentos de concreto com absorção de água baixa e resistentes a compressão; usadas como suplementos alimentares que auxiliam no combate e prevenção da osteoporose; como carga mineral em polímeros, na fabricação de materiais compósitos ecologicamente mais interessantes e com propriedades mecânicas e térmicas melhoradas, conferindo aos mesmos rigidezes e estabilidade térmica.
\end{abstract}

Palavras-chave: Resíduo da concha de molusco; Desequilíbrio ambiental; Aplicações; Sustentabilidade.

\begin{abstract}
Brazil is a major producer of bivalve molluscs in Latin America. Malacoculture activity is recognized worldwide as an important alternative for generating jobs, income and food, which has contributed to the establishment of traditional communities in their places of origin in a more sustainable way. However, the high densities of bivalve molluscs produced by cultivation can cause environmental impacts causing imbalances in ecosystems, due to a greater production of solid waste from the shells, which originate from the removal of molluscs from the interior of the shells. The objective of this article is to present a bibliographic review based on the current literature on the morphological structure of the mollusk shell and the possible applications of its residues. The outer shell structure is colored (periostracum), composed of an intermediate layer with compressed aragonite plates with a thickness of about $0.5 \mu \mathrm{m}$, embedded in a thin organic matrix and by another calcified layer containing calcite needles of various lengths and diameters., and by a nacreous inner layer that is also calcareous. Clam shells are mainly made up of calcium carbonate $(\mathrm{CaCO} 3)$. Therefore, shell residues have been used in the manufacture of concrete blocks and pavements with low water absorption and resistance to compression; used as food supplements that help fight and prevent osteoporosis; as a mineral filler in polymers, in the manufacture of ecologically more interesting composite materials with improved mechanical and thermal properties, giving them rigidity and thermal stability.
\end{abstract}

Keywords: Shellfish residue; Environmental imbalance; Applications; Sustainability.

\section{Resumen}

Brasil es un importante productor de moluscos bivalvos en América Latina. La actividad de la malacocultura es reconocida a nivel mundial como una importante alternativa para la generación de empleos, ingresos y alimentos, lo que ha contribuido al establecimiento de comunidades tradicionales en sus lugares de origen de una manera más sostenible. Sin embargo, las altas densidades de moluscos bivalvos que produce el cultivo pueden causar impactos ambientales provocando desequilibrios en los ecosistemas, debido a una mayor producción de desechos sólidos de las conchas, que se originan por la remoción de moluscos del interior de las conchas. El objetivo de este artículo es 
presentar una revisión bibliográfica basada en la literatura actual sobre la estructura morfológica de la concha de los moluscos y las posibles aplicaciones de sus residuos. La estructura de la capa externa es coloreada (periostracum), compuesta por una capa intermedia con placas de aragonito comprimido con un espesor de aproximadamente $0,5 \mu \mathrm{m}$, incrustadas en una fina matriz orgánica y por otra capa calcificada que contiene agujas de calcita de varias longitudes y diámetros, y por una capa interior nacarada que también es calcárea. Las conchas de almeja se componen principalmente de carbonato de calcio $(\mathrm{CaCO} 3)$. Por ello, los residuos de cáscara se han utilizado en la fabricación de bloques y pavimentos de hormigón con baja absorción de agua y resistencia a la compresión; utilizados como complementos alimenticios que ayudan a combatir y prevenir la osteoporosis; como carga mineral en polímeros, en la fabricación de materiales compuestos ecológicamente más interesantes con propiedades mecánicas y térmicas mejoradas, otorgándoles rigidez y estabilidad térmica.

Palabras clave: Residuos de mariscos; Desequilibrio ambiental; Aplicaciones; Sustentabilidade.

\section{Introdução}

A pesca artesanal de mariscos constitui uma atividade de fundamental importância para a redução da pobreza e fornecimento de alimento, além da geração de renda para comunidades costeiras. No entanto, esta atividade gera impactos ao meio ambiente devido ao acúmulo dos resíduos das conchas com a retirada do molusco de seu interior. O processo de beneficiamento gera uma grande quantidade desses resíduos, conforme mostra a Figura 1, em região próxima as margens do Rio Paraíba-PB. Após a extração do molusco, o descarte das conchas em ambiente de mangue ou ribeirinho, em grande quantidade, provoca assoreamento de grandes áreas e consequente desequilíbrio ambiental. Se descartadas em terrenos baldios, podem propiciar o aparecimento de animais e insetos transmissores de doença que se alimentam da matéria orgânica ainda presente nos resíduos das conchas. Além da decomposição da parte orgânica gerar mau cheiro, também pode ocorrer eventualmente o contato de banhistas com as conchas cortantes.

Figura 1. Resíduos de conchas de moluscos descartados próximos às margens do Rio Paraíba-PB.

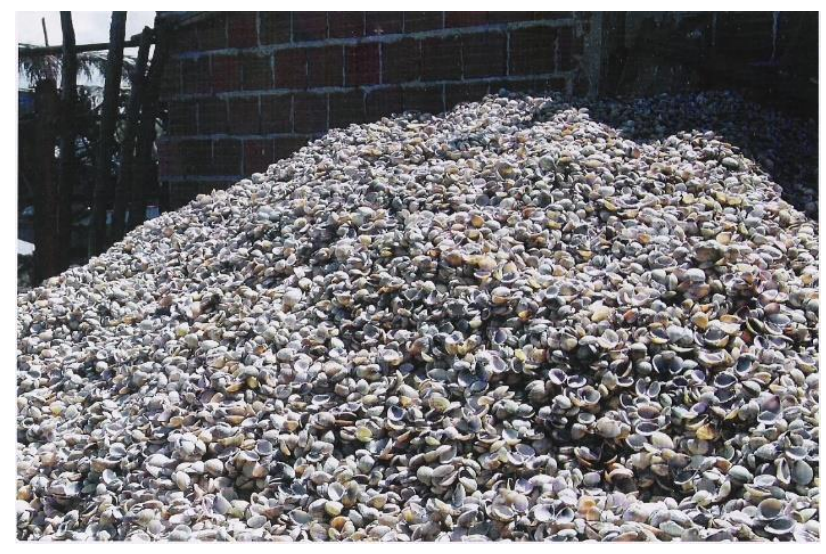

Fonte: Dados da Pesquisa.

A exploração de recursos marinhos faz parte das atividades das comunidades litorâneas. Dentre os recursos explorados, os mariscos habitam os sedimentos de planícies de maré adjacentes a manguezais. Por exemplo, na região costeira do Rio Paraíba-Brasil se desenvolve atividade de subsistência, de modo que os moluscos extraídos das conchas constituem uma expressiva fonte de renda para as comunidades tradicionais da zona costeira. Isto mostra por sua vez a importância dessa atividade na economia local das comunidades ribeirinhas. Os mariscos bivalves são abundantes nos bancos aluviais do Rio Paraíba. Esses são espécies comprimidas lateralmente e o corpo mole protegido por uma concha rígida constituída por duas válvulas - é um importante recurso explorado, com métodos rudimentares de extração. Assim existe uma carência de medidas de manejo que garantam o uso sustentável deste recurso (Bezerra et al.,2011). 
Em todo o mundo, as conchas de moluscos têm sido descartadas em aterros que tendem a causar danos ambientais pois sua decomposição produz amônia, sulfeto de hidrogênio e aminas com odores, além de cheirar mal e atrair camundongos, ratos, moscas e insetos (Li et al., 2012). A National Research Council dos Estados Unidos, revelou os principais impactos ambientais da malacocultura, os quais são: distúrbios das comunidades naturais de fitoplâncton, deterioração da qualidade da água devido à acumulação de dejetos e introdução de espécies que competem com as já existentes ou que transmitem doenças (Santanna, 2007). Na Coréia, desde os anos 80 são desenvolvidas pesquisas para utilização das conchas de ostras, devido à grande produtividade neste país. Eles pesquisaram a possibilidade de substituir os agregados na fabricação de cimento pelas conchas de ostras moídas, misturando estas com areia. A mistura foi considerada eficiente e uma boa alternativa em casos de escassez de areia (Yoon et al., 2003).

O objetivo desse trabalho é apresentar uma revisão sobre a estrutura morfológica das conchas e também mostrar a potencialidade dos resíduos das conchas como um material de utilidade em diversas aplicações de modo a colaborar com a mitigação de problemas ambientais causados pelo seu descarte em locais inadequados.

\section{Metodologia}

\section{Materiais}

Artigos e trabalhos científicos foram escolhidos utilizando bases de pesquisas como periódicos da Capes, sciencedirect.com, Google Acadêmico. Através dessa análise de trabalhos científicos procurou-se identificar e capturar as informações de cada estudo a fim de detalhar cada item a ser abordadas, com as informações contidas nesses estudos.

\section{Métodos}

A metodologia aplicada consistiu de modo geral na revisão, avaliação e identificação dos conteúdos a serem sintetizados. Uma pesquisa de campo foi realizada pelas autoras na Associação dos Marisqueiros do bairro Renascer em Cabedelo - PB, afim de melhor aquisição de conhecimentos para a escrita do presente trabalho. Seleção das fontes e trabalhos científicos; avaliação dos estudos; extração e síntese dos dados revisados e por fim discussão e interpretação dos dados.

\section{Relato de Caso}

Os dados apresentados a seguir foram obtidos a partir da revisão em diversos artigos provenientes da literatura vigente. Sendo estes apresentados e discutidos a começar pelas informações da constituição química e morfológica das conchas dos moluscos, seguida da apreciação de aplicações que podem ser sugeridas afim de mitigar problemas ambientais, os quais podem ser causados pela influência do descarte dos resíduos das conchas em ambientes pouco adequados para esta finalidade.

\section{Discussão}

\subsection{Conchas de moluscos}

A carne dos moluscos é valorizada na dieta humana por causa de seu sabor, textura e nutrientes. Já a concha de molusco causa problemas ambientais, pois é difícil descartar de maneira correta, transformando-se em resíduos, levando ao desgaste ambiental devido ao odor desagradável, assoreamento de rios, encostas, e desagradável aparência. A concha contém cerca de 95\% de carbonato de cálcio na forma de aragonita e calcita (Figura 2a) e 5\% de materiais orgânicos, como glicoproteínas, polissacarídeos, glicosaminoglicanos, quitina e outras proteínas. A maior parte dos materiais orgânicos é intercristalina, e, em menor grau, intracristalino (Figura 2b) (De Paula \& Silveira, 2003; Bielefeld et al.,1992). 
A concha de carbonato de cálcio, que protege os tecidos moles do molusco, constitui um excelente modelo para estudar o processo de formação biomineral e sua evolução. Todas as estruturas em camadas dessas conchas são extracelulares, que resultam da atividade secretora de um órgão evolutivo homólogo conhecido como manto, este secreta o Nácar. Em suma, o epitélio do manto expulsa os precursores iônicos da casca mineral juntamente com uma matriz orgânica extracelular que é incorporada no Nácar e envolve os cristais de $\mathrm{CaCO}_{3}$ nascentes durante o crescimento da concha (De Paula et al.,2005).

As conchas são formadas por nácar, uma mistura orgânica na forma de folhas finas (Figura 2c), descrita como segue: (1) uma camada proteica mais externa chamada perióstraco, frequentemente colorida; (2 e 3) uma capa intermédia de calcite ou aragonite (camada prismática mediana, com células impregnadas de cristais de carbonato de cálcio); (4) e por último uma camada de carbonato de cálcio $\left(\mathrm{CaCO}_{3}\right)$ cristalizado; (5) mais internamente, uma camada nacarada também calcária, geralmente, mais lisa e brilhante. As células da borda do manto secretam a camada prismática, enquanto as células da superfície produzem a camada nacarada. Isso faz com que a concha cresça simultaneamente em diâmetro e espessura (Mann, S.,2001; Silvia, M. et al.,2004). O nácar está entre os biomateriais mineralizados mais estudados (Mann, 2001). O nácar é secretado por células ectodérmicas do manto de certas espécies de moluscos. Muitos autores o consideram como o modelo de referência para compreender em escalas micro e nano como os moluscos controlam a deposição regular de cristais de carbonato de cálcio (Figura 2b). Além disso, o nácar é a camada interna calcificada existente em vários moluscos (Checa et al., 2009a).

Os cristais individuais de cada camada diferem na sua forma e orientação. A camada nacarada madura consiste na sobreposição de placas aragoníticas comprimidas de cerca de $0,5 \mu \mathrm{m}$ de espessura, incorporado em uma fina matriz orgânica periférica (Nudelman et al., 2008; Weiss, 2010). Os prismas que quase sempre estão associados ao nácar, são compostos de calcíticos ou aragoníticos semelhantes as estruturas de agulhas de vários comprimentos (Figura 2e) e diâmetros que sempre constituem a camada externa calcificada de conchas, e que crescem para dentro pelo acréscimo de unidades de cristal no interior da superfície do periósteo (Marin et al., 2007). Os prismas individuais são empilhados juntos em uma camada orgânica insolúvel e hidrofóbica, que forma uma estrutura em forma de favo de mel. Eles também incluem uma leve fração orgânica intracristalina (Rousseau et al.,2005). O nácar deposita-se de forma contínua na superfície interna da concha, e essa capa nacarada é também conhecida como madrepérola (Figura 2f). Estes processos proporcionam ao molusco, um mecanismo de defesa contra organismos parasitas (Gilbert et al, 2008). 
Figura 2. Microscopia eletrônica de varredura (MEV):a) amostra de Calcita; b) Cristais poliedricos de Aragonita na matriz orgânica e Cristais romboedros de Calcita em contato com matriz orgânica c) Secção transversal da concha; d) Camada nacarada e) MEV da secção transversal da concha de marisco (ampliação da região 2 da letra c); f) Camada nacarada exibindo tabletes de $\mathrm{CaCO}_{3}$ poligonais.
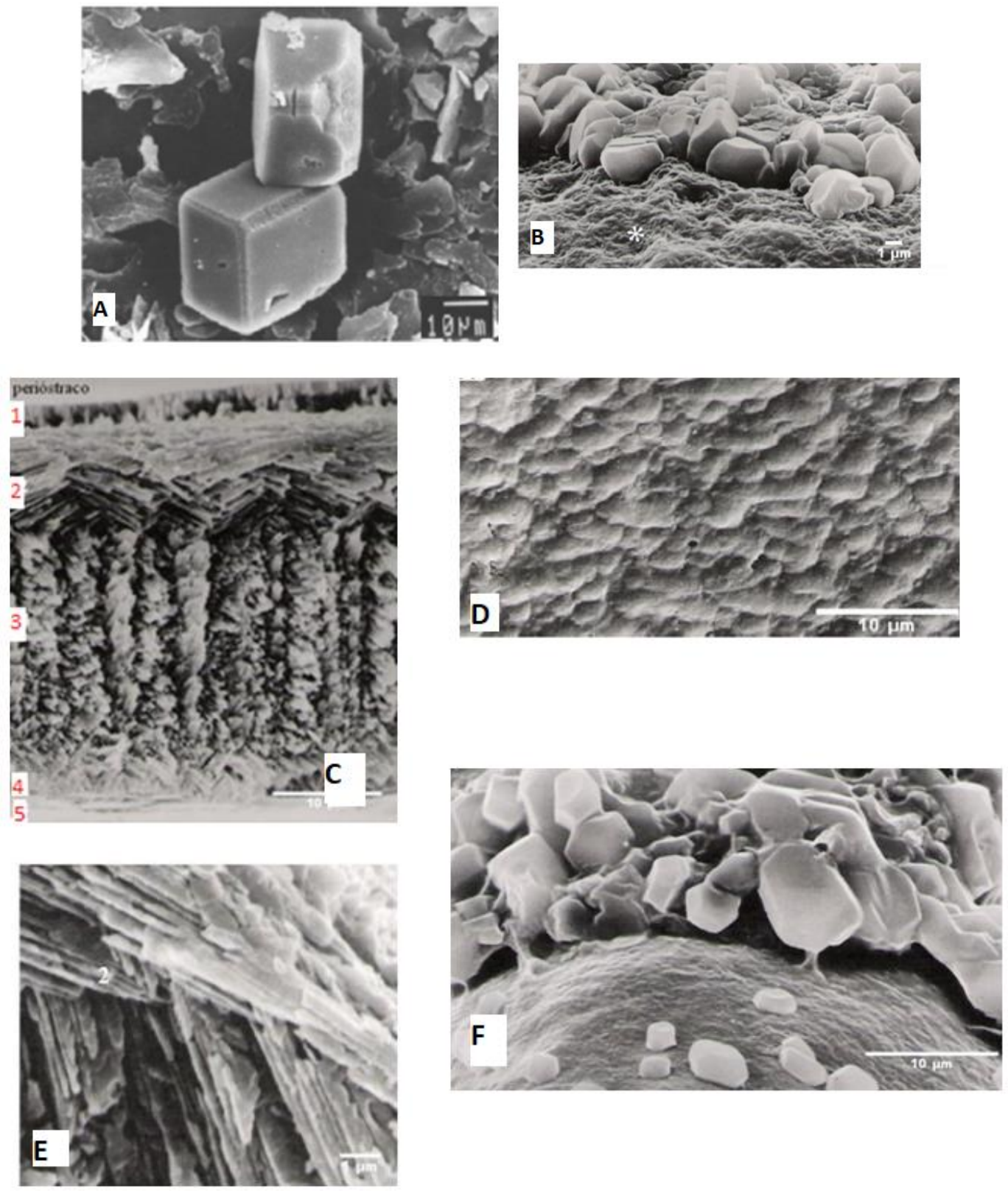

Fonte: Adaptado de De Paula, S. M. (2006) com a prévia permissão.

Mesmo em pequenas quantidades, as proteínas da matriz da concha que estão incorporadas nas camadas calcificadas das conchas dos moluscos desempenham um papel essencial no controle da síntese biomineral e no aumento de suas propriedades mecânicas (Hare et al, 1965). As conchas de moluscos são biocompósitos minerais orgânicos fascinantes com alto desempenho mecânico. Com apenas $1-5 \%$ em peso de material orgânico, um controle de crescimento de tamanho nanométrico é alcançado o que aumenta a resistência da casca consideravelmente em comparação com o mineral não biogênico (Cariolou \& Morse 1988). A concha do molusco tem baixo teor de proteína e gordura de acordo com Yusuff e Oseni (2004). A 
principal função da concha é a proteção do molusco. Para tanto, em sua constituição também tem a presença de alto teor de carboidratos, que ao contrário da proteína e da gordura, tem fortes propriedades de ligação que não se decompõem facilmente na presença de elementos meteorológicos diversos. Isso acontece pois os constituintes orgânicos da concha são polissacarídeos e glicoproteínas. Com esse cenário composicional, as conchas podem ser adicionadas à alimentação animal como fonte de energia (Hodasi, 1982). A matriz orgânica representa apenas uma pequena parte do peso da casca de $\mathrm{CaCO}_{3}$, porém sabe-se que é essencial para o controle da formação desse biomineral (Mann, 1988). Uma análise em Microscópio Eletrônico de Varredura (MEV) realizada por Li et al (2012) é similar a morfologia observada por De Paula (2006), conforme Figura 3, possibilitando ver a organização da camada de aragonita com estrutura regular e finas folhas de camada orgânica alternadas formando uma arquitetura compacta (Li et al.,2012).

Figura 3. Secção transversal da concha.

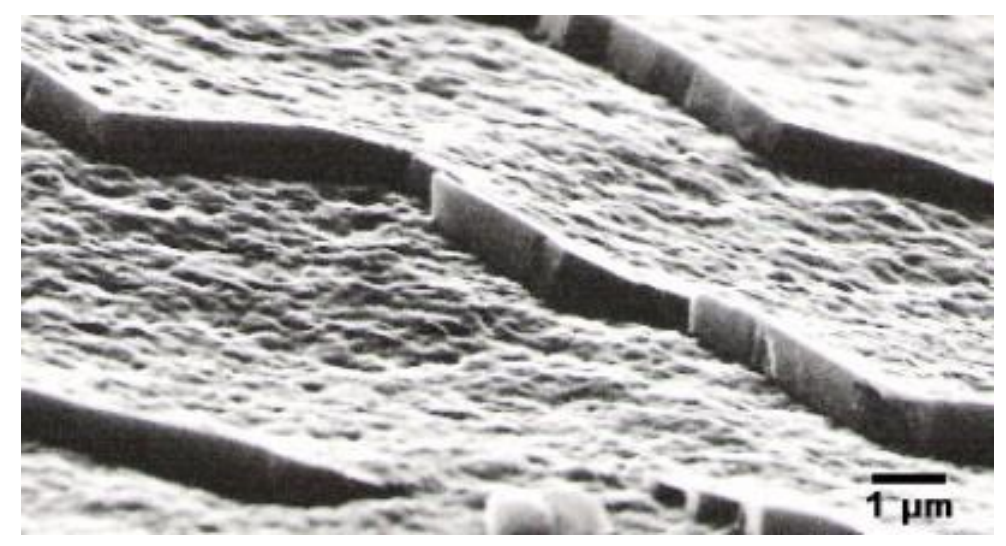

Fonte: Adaptado de De Paula, S. M. (2006) com a prévia permissão.

Ademolu et al, (2007) observaram que a composição da concha muda conforme resultado da mudança na química da água. Os sedimentos marinhos anóxicos subtidais (condição de baixa oxigenação) contêm alta quantidade de sulfato solúvel $\left(\mathrm{H}_{2} \mathrm{~S}\right)$ e ácido sulfato lábil ( $\left.\mathrm{FeS}\right)$. Além disso, o $\mathrm{Ca}^{2+}$ foi significativamente maior do que outros íons presentes nas conchas. Uma análise mineral das conchas dos moluscos de vários locais da Nigéria mostrou que havia diferenças significativas na concentração de íons em todas as espécies de moluscos. Observou-se que as amêijoas, espécies de moluscos bivalves da ordem Veneroida, tiveram significativamente maior quantidade de $\mathrm{Fe}^{2+} \mathrm{e} \mathrm{Ca}^{2+}$ do que outras espécies de moluscos (Ademolu et al, 2007). Este resultado pode provavelmente ser devido à sua localização, ou seja, em ambiente marinho, enquanto outros moluscos são terrestres. Isso pode explicar o uso das conchas como abrasivos, e agentes de escovagem. Estudos relatam o uso da concha na coagulação do sangue, onde envolve a conversão de fibrinogênio em fibrina, formando uma fina rede que cobre a ferida (Agbelusi \& Ejidike, 1992). As conchas também apresentaram baixa concentração de $\mathrm{Na}^{+}$, que é coerente com a concentração encontrada na parte comestível, que também tem sido relatada ser baixa (Ademolu et al, 2007; Idowu et al., 2008). Assim, as conchas podem ser incluídas na pecuária e na dieta do homem sem medo de complicações decorrentes de alta ingestão de $\mathrm{Na}^{+}$.

\subsection{Aplicações}

Como já mencionado anteriormente as conchas são ricas em carbonato de cálcio, podendo assim serem reutilizadas para agricultura e indústria. O carbonato de cálcio é usado em mármore compacto para pavimentos e revestimentos, adubos, indústria de cerâmica, tijolos, tintas, carga de polímeros, entre outros (Boicko et al, 2004). Também é possível sua utilização para correção de acidez de solos, através da prática da calagem, a qual neutraliza a ação das substâncias causadoras da acidez 
no solo, muito característica dos solos do Distrito Federal-Brasil, como exemplo conchas de moluscos também foram usadas como fonte de suplementos de cálcio para vacas em lactação (Finkelstein et al, 1993). Agbelusi e Ejidike (1992) relataram que a concha de caracol foi usada não apenas como fonte de cálcio para animais de fazenda, mas como um ingrediente importante em Medicina tradicional (Agbelusi \& Ejidike, 1992).

\subsection{Aplicações Industriais}

Existem diversas formas de reutilizar os resíduos das conchas, pela sua composição já citada. Os produtos que podem se utilizar desta fonte de $\mathrm{CaCO}_{3}$ são: cal virgem, cal hidratada, carga em polímeros, construções de estradas, pasta de papel (substituindo em parte a matéria-prima vegetal), espumas de polietileno, produção de talco, produção de vidros, indústria do cimento, produção de vernizes e borrachas, impermeabilização de lagoas, selagem de lixeiras (material impermeabilizante) e medicamentos. A adição de carbonato de cálcio resulta em produtos de boas propriedades mecânicas e com boa possibilidade de pigmentação, podendo agir como agente nucleante, aumentando por exemplo a durabilidade de compósitos poliméricos. O carbonato de cálcio entra na composição de perfis, tubos para água e pisos, revestimentos, fios e cabos elétricos. Pode ser utilizado na fabricação de tubos de PVC, pois é necessária a incorporação de aditivos para que o policloreto de vinila (PVC) passe de resina plástica para o produto final. Esses aditivos tem a finalidade de abaixar o custo ou melhorar determinada propriedade dependendo da utilização do PVC pronto (Boicko et al, 2004; Finkelstein et al, 1993).

\subsection{Aplicações na Construção Civil}

Estudos realizados por pesquisadores da Universidade do Sul de Santa Catarina (UNISUL) mostraram a viabilidade do uso de cascas de ostras e mexilhões na fabricação de blocos de concreto e de blocos para a pavimentação. As cascas dos moluscos passaram por um processo de lavagem, depois foram secas ao sol para posteriormente serem trituradas em moinho. Após a trituração as cascas foram misturadas aos demais componentes da fabricação de blocos e pavimentos de concreto. Esses blocos passaram por testes de absorção de água e resistência à compressão. Os resultados obtidos pela pesquisa mostraram que estes produtos são altamente viáveis se encaixando nas normas da ABNT. Esta seria mais uma alternativa para o uso dos resíduos provenientes da malacocultura (Batista, 2009). Na Coréia do Sul (Ásia) foi estudada a possibilidade da substituição de agregados miúdos por conchas de ostras moídas na fabricação de argamassa, principalmente para usos mais viáveis como materiais para preenchimento na construção de diques. Esta foi considerada uma boa alternativa em casos de pouca disponibilidade de areia (Yoon et al.,2003).

Bezerra et al. (2011) realizou estudos adicionando resíduos de concha de moluscos ao cimento Portland para atuar como carga de enchimento, e também obter um calcário que pode ser usado em tintas, pois o material é composto grade parte de carbonato de cálcio (Bezerra et al.,2011). Os resultados mostraram que devido à baixa concentração de sílica nas conchas, não houve a reação pozolânica, porém o resíduo da concha pode ser usado com efeito de enchimento no cimento. Ainda, de acordo com Bezerra et al (2011), a concentração em massa de 10\% de resíduo de concha, contribuiu para aumentar a resistência a compressão nas argamassas testadas, atuando como enchimento para o Cimento Portland (Bezerra et al.,2011).

\subsection{Aplicações na Indústria farmacêutica}

A indústria farmacêutica também tem se beneficiado do carbonato de cálcio extraído das conchas de moluscos. Um exemplo, são os suplementos alimentares que auxiliam no combate e prevenção da osteoporose. Cientistas americanos mostraram que no combate a tensão pré-menstrual, o cálcio da concha de ostra reduziu em 50\% os sintomas, diminuindo consideravelmente a depressão e as cólicas (Santanna, 2007). Estudos realizados na Flórida (EUA) e Coréia do Sul também revelam quem as conchas de ostras, após serem pirolisadas a uma temperatura de $750^{\circ} \mathrm{C}$ durante 1 hora em uma atmosfera de 
nitrogênio, transformam-se em um produto com eficiência maior que 98\% para remoção de fosfatos em águas residuárias, sendo uma importante estratégia para o controle da eutrofização de águas (Kwon et al, 2003).

\subsection{Aplicação como carga em compósitos poliméricos}

Cargas de baixo custo são adicionados aos polímeros para reduzir custos e melhorar suas propriedades. Entre elas, uma carga inorgânica amplamente utilizada é o carbonato de cálcio $\left(\mathrm{CaCO}_{3}\right)$ (Melo et al.,2019), sendo também o principal constituinte das conchas bivalves e matéria-prima para diversos produtos. As conchas são abundantes e são subprodutos descartados que podem servir como substitutos potenciais para o calcário derivado de rochas sedimentares. É importante que se busquem formas corretas de se descartar, e se possível, reutilizar os resíduos sólidos ou líquidos, gerados nas atividades de malacocultura.

Bocchese (2008) destaca que para se reutilizar os resíduos da pescagem de mariscos é necessário que se elimine a matéria orgânica e possíveis organismos que estejam aderidos à superfície das conchas (Bocchese, 2008). Uma forma de processar as conchas para obtenção do carbonato de cálcio seria a moagem a úmido, seguida de tratamento térmico (400 $500^{\circ} \mathrm{C}$ ), para eliminação da água da moagem e retirada do substrato orgânico presente entre as camadas de aragonita das conchas, como mostrado na Figura 6. Esta forma de processamento tem a desvantagem de ser muito onerosa, dificultando a utilização desses resíduos como matéria-prima renovável.

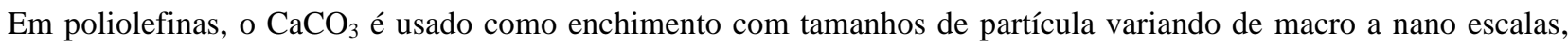
devido a conferir tenacidade e rigidez aos compósitos poliméricos. Polietileno de Alta densidade (HDPE) com cargas particuladas é de grande interesse em aplicações industriais devido a facilidade de processamento, e pelo bom desempenho com diferentes tipos de cargas naturais e sintéticas. Os efeitos das adições de cargas inorgânicas nas propriedades mecânicas e propriedades térmicas de matrizes poliméricas demonstram resultados positivos (Shao-Yun Fu et al., 2008; Bartezak et al., 1999).

Li (2012) avaliaram compósitos de Polipropileno (PP) com resíduos de concha e com carbonato de cálcio comercial (Li et al.,2012). Eles ressaltaram que as partículas da bio-aragonita natural (SS) contendo uma pequena quantidade de matéria orgânica apresentou maior compatibilidade com a matriz de PP, com bons resultados de módulo de Young e tensão de escoamento superiores aos compósitos com $\mathrm{CaCO}_{3}$ comercial, fato esse explicado pela boa dispersão de SS na matriz. Além disso, a dispersão de SS na matriz polimérica promoveu a nucleação heterogênea da matriz de PP.

Estudos em compósitos de polietileno de alta densidade (PEAD) com resíduos de concha de molusco com variação de tamanho de partícula e concentrações baixas de 2, 5 e $8 \%$ em peso revelaram que o teor de $8 \%$ em peso com tamanhos de partículas variados, não modificou significativamente o MFI dos compósitos (Melo et al.,2019). No entanto, maior tamanho de partículas dos resíduos da concha e em maiores concentrações até 20\% nos compósitos de PEAD (Firmino et al., 2017) agiram como falhas, e ao deslizarem umas sobre as outras, reduziram a taxa de fluxo desses compósitos em comparação a daqueles com menores tamanhos de partículas e menos concentrações (Melo et al.,2019). O resultado obtido por Munusamy et al. (Munusamy et al., 2019) também revelou um aumento da viscosidade do polietileno de baixa densidade (PEBD) com a adição de $20 \%$ de concha em acordo com (Firmino et al., 2017). Melo et al. [36] observaram ao calcinar o resíduo da concha a $500{ }^{\circ} \mathrm{C}$ a diminuição do tamanho das partículas, e a adição de $20 \%$ em peso ao Polipropileno reciclado não resultou em mudança no MFI da matriz; por outro lado ao modificar o resíduo calcinado com óleo da castanha de caju ou com polietileno glicol promoveu considerável aumento da viscosidade do compósito devido a interação molecular entre as fases. Portanto, o tamanho das partículas da concha ou a modificação da sua superfície têm efeito na processabilidade das matrizes termoplásticas.

De acordo com Melo et al. (2019) (Melo et al.,2019), a temperatura de cristalização inicial dos compósitos não mostrou mudanças em relação ao PEAD, permanecendo em $114^{\circ} \mathrm{C}$ para cada concentração avaliada, coerente com Firmino et 
al. (Firmino et al., 2017) que observaram apenas mudanças na temperatura de fusão, comparando PEAD e os compósitos, fato esse atribuído a matriz pura, em que seus cristais são mais alinhados em comparação aos dos compósitos. A cristalinidade do PEAD também não foi alterada com adição do resíduo da concha "in natura" (Melo et al.,2019; Firmino et al., 2017) e nem calcinado (Melo et al, 2021). Outros pesquisadores trabalhando com compósitos a base de concha com o PEBD (Munusamy et al., 2019) e com o PP (Bartezak et al., 1999) obtiveram resultados positivos de aumento da cristalinidade dessas matrizes, sugerindo a atuação do resíduo da concha como agente nucleante efetivo. Com respeito a estabilidade térmica dos compósitos poliméricos baseados nesse resíduo de concha, vários autores têm reportado aumento na temperatura de degradação térmica (Bartezak et al., 1999; Yao et al., 2013), esse comportamento térmico é similar ao já observado por outros (Luyt et al., 2009; Manish et al., 2012) que trabalharam com compósitos poliméricos contendo cargas minerais. De modo que a melhoria na estabilidade térmica desses compósitos tem sido atribuída ao impedimento da difusão de produtos voláteis dentro do polímero devido a formação de carbonáceos, que atuam como uma barreira física, impedindo a difusão de calor e consequente degradação (Luyt et al., 2009; Manish et al., 2012).

A modificação dos termoplásticos com resíduos de conchas tem aumentado as propriedades mecânicas dos compósitos. É conhecido da literatura que o módulo sob tração de uma matriz polimérica melhora com a adição de partícula rígida porque sua rigidez é geralmente muito maior do que a da matriz (Shao-Yun Fu et al., 2008). Em termos do tratamento superficial no resíduo da concha, para o módulo de elasticidade sob flexão tem efeito positivo (Bartezak et al., 1999) enquanto para o módulo sob tração melhores resultados são reportados para a concha sem tratamento (Munusamy et al., 2019; Melo et al., 2021; Pardo et al., 2010). A resistência ao impacto pode aumentar com a modificação superficial do resíduo (Melo et al., 2021) ou até mesmo ter comportamento indiferente ao tratamento (Zhitong et al., 2014). Já a tensão de escoamento é mais sensível à modificação superficial devido aumentar a interação entre os constituintes e consequente a adesão, conduzindo aos mecanismos que retardam a fratura. É bem reportado que a presença de aglomerados das partículas inorgânicas reduz as propriedades dos compósitos (Feng et al., 2014; Essabir et al., 2017), justamente por impedir a ação de mecanismos que previnem a fratura em tensões mais baixas. Em outras situações, a presença dos resíduos da concha não altera as propriedades mecânicas das matrizes poliméricas (Li et al.,2012; Melo et al.,2019). No entanto, a utilização desse poluente em compósitos poliméricos como benefício a diminuição do impacto ambiental proveniente das atividades de malacocultura.

\section{Conclusão}

Os problemas ambientais causados pelo resíduo da concha, tornam a sua utilização emergente afim de mitigar a poluição causada pelo seu descarte inadequado. A variedade de aplicações apresentadas na indústria como produção de vidros, produção de borrachas, assim como na indústria de construção, em argamassas, pisos, revestimentos; na indústria farmacêutica em tratamentos como por exemplo da osteoporose, demostra a gama de possibilidades de utilização do resíduo da concha de molusco. Nos compósitos poliméricos, se observou aumento nas suas propriedades mecânicas devido a capacidade desse resíduo em atuar como reforço. A concha tem uma microestrutura calcárea composta por placas micrométricas de aragonita com espessa nanométrica, e calcita com formato de agulha em vários tamanhos, as quais são organizadas de uma forma altamente precisa e adaptativa. Desse modo, tem tido aplicação surpreendente em compósitos poliméricos, proporcionando aumento no módulo de elasticidade tanto sob tração quanto sob flexão, na tensão de escoamento e resistência ao impacto, promovendo assim rigidez e estabilidade térmica aos mesmos. Já o índice de fluidez do polímero tendeu a diminuir com o aumento do tamanho das partículas do resíduo da concha e da sua concentração. Portanto, o uso do resíduo em quantidades até 20\% em peso e com tamanho de partículas de tamanho micrométrico impulsiona a produção de compósitos poliméricos com características mecânicas e térmicas apreciáveis para aplicações diversas ecologicamente atraentes. 
Estudos futuros podem ser pautados em pesquisas agregando o resíduo de concha de molusco em matrizes poliméricas ou cerâmica, variando os percentuais, com o objetivo de melhorar as propriedades e ampliar as aplicações dessas combinações de materiais. Para tanto, é preciso ter atenção com respeito à distribuição de tamanho das partículas do resíduo da concha. Como também realizar tratamentos superficiais, em se tratando de compósitos poliméricos, afim de melhorar a dispersão das partículas e fortalecer a adesão interfacial entre os componentes para obter produtos com resistência mecânica e custo de produção apreciáveis.

\section{Agradecimentos}

Os autores agradecem a Coordenação de Aperfeiçoamento de Pessoal de Nível Superior CAPES pela concessão de bolsa de estudos a aluna. A UFPB pela infraestrutura dos laboratórios onde foram executados os trabalhos científicos, dando suporte para a escrita do artigo, assim como pela formação e capacitação fornecida pela mesma.

\section{Referências}

Ademolu, K. O., Idowu, A. B., Mafiana, C. F., \& Osinowo, O. A. (2007). Performance, proximate and minerals analysis of African giant land snail (Archachatina marginata) fed different nitrogen sources. Tropical Veterinarian, 25(4), 124-131.

Agbelusi, E. A., \& Ejidike, B. N. (1992). Utilization of the Giant African Land Snail, Archachatina marginata in the humid area of Nigeria. Journal of Tropical Agriculture, 69, 88-92.

Bartezak, Z., A. S.A., Cohen, R. E., \& Weinberg, M. (1999). Toughness mechanism in semi-crystalline polymer blends; II. High-density polyethylene toughened with calcium carbonate filler particles. Elsevier Science Ltd. p. 2347-2365.

Batista, B. B., Silva, H. R. T., Egert, P., Marcondes, L. F. T., \& Santos, M. V. (2009). Bloco Verde - Reaproveitamento de Resíduos da Construção Civil e de Conchas de Ostras e Mariscos. $1^{\circ}$ Congresso Internacional de Tecnologias para o Meio Ambiente, Bento Gonçalves, RS, Brasil.

Bezerra, U.T. A. ; Almeida, F. L. P; Silva, L. B; Barbosa, N. P; Passos, T. A, \& Cavalcante, D. G. L. (2011). Production of Filler Aggregate from Waste of Bivalves Molluscs Shells. Jornal of Civil Engineering and Architecture, 5: 363-367.

Bielefeld U, Zierold K., Korje K. H., \& Becker W (1992) Calcium localization in the shell-forming tissue of the freshwater snail, Biomphalaria glabrata: a comparative study of various methods for localizing calcium. Histochem J 24:927-938.

Bocchese, D. C. F. (2008). Eliminação de matéria orgânica de conchas de ostras por processo biológico. Repositório Institucional Universidade Federal de Santa Catarina, Florianopolis, SC.

Boicko, A. L., Hotza, D. \& Santanna, F. S. P. (2004). Utilização de Conchas Da Ostra Crassostrea gigas Como Carga Para Produtos de Policloreto de Vinila (PVC). IV Simpósio Internacional de Qualidade Ambiental.

Cariolou, M. A., \& Morse, D. (1988). Journal of Computational Physics B 157, 717.

Checa, A. G., Cartwright, J. H., \& Willinger, M. G. (2009a) The key role of the surface membrane in why gastropod nacre grows in towers. Proceedings of the National Academy of Sciences 106:38-43.

De Paula, S. M, \& Silveira, M. (2006). Uma abordagem de parâmetros de biomineralização em um sistema constituído por carbonato de cálcio. Biblioteca Digital USP, Brasil.

Essabir, H., Bensalah, M. Q., Rodrigue, D., Bouhfid, R., \& Qaiss, A. K. (2017) Acomparison between bio- and mineral calcium carbonate onthe properties of polypropylene composites. Construction and Building Materials; 134:549-55.

Finkelstein, A. D., Wohlt, J. E., \& Emmanuele, S. M. (1993). Composition and nutritive value of ground sea clam shells as calcium supplements for lactating Holsten cows. Journal of Dairy Science, 76(2), 582-589.

Firmino, H. C. T., Chagas, T. F., Melo, P. M. A., \& Silva, L. B. (2017). Caracterização de compósitos particulados de polietileno de alta densidade/pó de concha de molusco. Matéria (Rio J.), 22(4), 1-12.

Feng, Y., Ashok, B., Madhukar, K., Zhang, J., Zhang, J., \& Reddy, K. O. (2014). Preparation and characterization of polypropylenecarbonate bio-filler (eggshell powder) composite films. International Journal of Polymer Analysis and Characterization 2014:19:637-47.

Gilbert, P. U., Metzler, R. A., Zhou, D., Scholl, A., Doran, A., Young, A., Kunz, M., Tamura, N., \& Coppersmith, S. N. (2008). Gradual ordering in red abalone nacre. Journal of the American Chemical Society 130:17519-17527.

Hare, P. E., \& Abelson, P. H. (1965). Year Book, Carnegie Inst. Washington, 65, 223.

Hodasi, J. K. N. (1982). The effect of different light regimes on the behavior and biology of Achatina achatina. Journal of Molluscan Studies, 48, 1-7. 
Idowu, A. B., Somide, O. M., \& Ademolu, K. O. (2008). Comparative Analysis of the chemical composition of the haemolymph, flesh and the microflora content of the guts of some African land snails in Abeokuta, Nigeria. Tropical Veterinarian, 26(1\&2), 20-29.

Kwon, H., Lee, C. W., Jun, B. S., Yun, J., Weon, S. Y., \& Koopman, B. (2003). Recycling waste oyster shells for eutrophication control. Resources, Conservation and Recycling, Masan, Coréia do Sul, 41, 75-82. https://projetoconchas.ufsc.br/upload/arquivos/1197924433.PDF

Li, H. Y., Tan, Y. Q., Zhang, L., Zhang, Y. X., Song, Y. H., Sim, Y., \& Xia, M. S. (2012). Bio-filler from waste shellfish shell: Preparation, characterization, and its effect on the mechanical properties on polypropylene composites. Journal of Hazardous Materials, 217-218, $256-262$.

Luyt, A. S., Dramicanin, M. D., Antic, Z., \& Djokovic, V. (2009). Morphology,mechanical and thermal properties of composites of polypropylene and nanostructured wollastonite filler. Polymer Testing 28:348-56.

Manish, K., Sharma, C. S., Pradeep, U., Vishal, V., Pandev, K. N., \& VijaiK. (2012) Calcium carbonate (CaCO3) nanoparticle filled polypropylene: effect of particle surface treatment onmechanical, thermal, and morphological performance of composites. Journal of Applied Polymer Science, 124:2649-56.

Mann S. (2001) Biomineralization: principles and concepts in bioinorganic materials chemistry. Oxford University Press, New York.

Mann, S. (1988). Molecular recognition in biomineralization. Nature 332:119-124.

Marin, F., Pokroy, B., Luquet, G., Layrolle, P., \& De Groot, K. (2007). Protein mapping of calcium carbonate biominerals by immunogold. Biomaterials 28:2368-2377.

Masaya, K., Naoki, H., Makoto, K., Arimitsu, U., \& Akane, O. (1997). Preparation and mechanical properties of polypropylene clayhybrids. Macromolecules, 30:6333-8.

Melo, P. M. A., Macêdo, O. B., Barbosa, G. P., Ueki, M. M., \& Silva, L. B. (2019). High-density polyethylene/mollusk shell-waste composites: effects of particle size and coupling agent on morphology, mechanical and thermal properties. Journal of Materials Research and Technology, 8(2) 1915-1925.

Melo, P. M. A., Macêdo, O. B., Barbosa, G. P., Santos, A. S. F., \& Silva, L. B. (2021). Reuse of Natural Waste to Improve the Thermal Stability, Stiffness, and Toughness of Postconsumer Polypropylene Composites. Journal of Polymers and the Environment; 29, 538-551. https://doi.org/10.1007/s10924-02001907-4

Munusamy, Y., Sethupathi, S., \& Choon, C. H. (2019). Potential use of waste cockle shell as filler for thermoplastic composite. Journal of Material Cycles and Waste Management, 21:1063.

Nudelman, F., Shimoni, E., Klein, E., Rousseau, M., Bourrat, X., Lopez, E., Addadi, L., \& Weiner, S. (2008). Forming nacreous layer of the shells of the bivalves Atrina rigida and Pinctada margaritifera: an environmental- and cryo-scanning electron microscopy study. Journal of Structural Biology 162:290300 .

Pardo, S. G., Bernal, C., Ares, A., Abad, M. J., \& Cano, J. (2010). Rheological, thermal, and mechanical characterization of Fly Ashthermoplastic composites with different coupling agents. Polymer Composite 31:1722.

Rousseau, M., Lopez, E., Stempfle, P., Brendle, M., Franke, L., Guette, A., Naslain, R., \& Bourrat, X. (2005). Multiscale structure of sheet nacre. Biomaterials 26:6254-6262.

Saminathan, K., Selvakumar, P., \& Bhatnagar, N. (2008). Fracture studiesof polypropylene/nanoclay composite. Part II: failure mechanism under fracture loads. Polymer Testing 27:453-8.

Santanna, F. S. P. (2007). Projeto Valorização dos resíduos da Maricultura. Sub-projeto: Soluções tecnológicas para aproveitamento de conchas de ostras. Universidade Federal de Santa Catarina, Florianópolis.

Shao-Yun Fu, X. Q. F., Bernd L., \& Yiu-Wing M. (2008). Effects of particle size, particle/matrix interface adhesion and particle loading on mechanical properties of particulate-polymer composites. Elsevier Composites: Part B 39. 933-961.

Zhitong, Y., Meishen,g X., Liugin, G., Tao, C., Haiyan, L., Ying, Y., \& Zheng, H. (2014). Mechanical and thermal properties of polypropylene (PP) composites filled with CaCO3and shell-waste derived bio-fillers. Fiber Polym 15:1278.

Yao, Z.T., Chen, T., Li, H.Y., Xia, M.S., Ye, Y. \& Zheng. (2013). Mechanical and thermal properties of polypropylene (PP) composites filled with modified shell waste. Journal of Hazardous Materials, 262, 212-217.

Yoon, Gil-Lim; Kim, Byung-Tak; Kim, Baeck-Oon; Han, Sang-Hun. (2003). Chemical-mechanical characteristics of crushed oyster-shell. Coastal \& Harbor Engineering Research Division, Korea Ocean Research \& Development Institute, 1270, Sadong, Ansan City, 425-744, South Korea.

Yusuff, A. A., \& Oseni, O. A. (2004). Nutritional value and functional properties of pond snail (Lymnae stagnalis). International Conference on Science \& National Development. Department of Chemistry, University of Agriculture, PMB 2240. Abeokuta, Nigeria.

Weiss, I. (2010). Jewels in Pearl. ChemBioChem 11:297-300. 